Fourth International Conference on Sustainable Construction Materials and Technologies http://www.claisse.info/Proceedings.htm

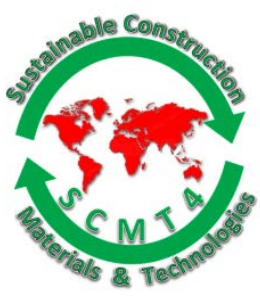

SCMT4

Las Vegas, USA, August 7-11, 2016

\title{
Stress Intensity Factors for Inclined External Surface Cracks in Pressurized Pipes
}

\author{
Guoyang Fu' ${ }^{1 \mathrm{a}}$, Chun-Qing Li ${ }^{1 \mathrm{~b}}$, Wei Yang ${ }^{2}$, Sujeeva Setunge ${ }^{1 \mathrm{c}}$ and Annan Zhou ${ }^{1 \mathrm{~d}}$ \\ ${ }^{1}$ School of Civil, Environmental and Chemical Engineering, RMIT University, Melbourne 3001, \\ Australia. ${ }^{1 a}$ Email: < guoyang.fu@rmit.edu.au> ${ }^{1 b}$ Email: $<$ chunqing.li@rmit.edu.au>, \\ ${ }^{1 c}$ Email: <sujeeva.setunge@rmit.edu.au>, ${ }^{1 d}$ Email: <annan.zhou@rmit.edu.au>. \\ ${ }^{2}$ School of Civil Engineering and Architecture, Wuhan University of Technology, Wuhan, 430070, \\ China.²Email: <missyangw@163.com>
}

\begin{abstract}
Inclined surface cracks may occur in pressurized pipes due to corrosion, poor manufacturing quality, complex loading systems or a combination of these factors. However, very few studies have been conducted on the stress intensity factors for inclined cracks in pipes. Due to the high failure rate of pipes with brittle failure, it is essential to thoroughly examine the causes of pipe failures. This paper is intended to study the stress intensity factors with mixed modes (Modes I, II and III) for inclined external surface cracks in pressurized pipes. A three dimensional finite element model meshed with mixed quadratic tetrahedron and hexahedron elements is employed. After verification of the finite element model, the influence coefficients of the stress intensity factors for a wide range of inclined external cracks in a pressurized pipe are obtained. Based on the numerical results, it was found that the influence coefficients of both mode I and equivalent stress intensity factors are symmetric with respect to the deepest point and decrease along the whole crack front with the increase of the inclination angle. In contrast, those of mode II and mode III reach the maximum when the inclination angle is $45^{\circ}$ and decrease when the angle increases or decreases from $45^{\circ}$. It was also observed that, the maximum mode I stress intensity factors of semi-elliptical cracks with high aspect ratios for different inclination angles all appear near the free surface, which is different from the semi-elliptical cracks with low aspect ratio. In addition, for given wall thickness to internal radius ratio and aspect ratio, the influence coefficients of all three modes stress intensity factors along the whole crack front for all inclination angles, increase with the increase of the relative depth.
\end{abstract}

\section{INTRODUCTION}

Pipelines are essential infrastructure that plays a significant role in a nation's economy and quality of life. Due to their long term service and exposure to corrosive environment, aging and deterioration of metal pipes have resulted in a very high rate of pipe failures. For instance, the failure rate of cast iron pipes can be as high as 39 bursts per $100 \mathrm{~km}$ per year in the Canada [Makar 2000]. An investigation on pipe failures reveals that most metal pipe failures are of fracture type, which is caused by the instable propagation of a crack or defect on the pipe surface [Marshall 2001]. Due to corrosion, poor manufacturing quality, complex loading systems or a combination of these factors, surface cracks are frequently observed in these cylindrical 
structures. These cracks occur in longitudinal, circumferential or inclined directions and have long been recognized as a major cause of potential failures for cylindrical structures. The consequence of pipe failures can be huge and devastating, resulting in massive disruption of daily life and great inconvenience, unexpected tilt or even collapse of nearby structures, widespread flooding and subsequent environmental pollution, etc. Therefore, it is of high importance to thoroughly examine the causes of pipe failures.

The cracks are generally considered to be semi-elliptical in shape and the centres are assumed to be located on the pipe surface [Lin and Smith 1997; 1998; Carpinteri and Brighenti 2000]. In order to determine the crack growth rates and predict the remaining life of the cracked pipe structures, it is essential to accurately estimate the stress intensity factors along the crack. So far, the fracture analyses of cylindrical structures have drawn the attention of many researchers and extensive studies have been carried out. A review of the available literature suggests that previous research mainly focuses on the study of either longitudinal or circumferential cracks in cylindrical structures.

The stress intensity factors of longitudinal cracks in pipes have been studied by a number of researchers. Using a nodal force method, Raju and Newman [1982] obtained the stress intensity factors for internal and external surface cracks with aspect ratios from 0.2 to 1.0. Then Wang and Lambert [1994] extend the work to include the aspect ratios smaller than 0.2 (0.05 and 0.1). Realizing that cracks with high aspect ratio $(a / c>1)$ may often appear for pitting corrosion of cast iron and steel pipes and propagation of fatigue induced cracks, Li and Yang [2012] determined the stress intensity factors for internal surface cracks in pipes with high aspect ratios. For circumferential cracks, Raju [1991] derived the stress intensity factors for surface cracks with cylinder radius to wall thickness ratio $R / t=1$ and 2 with crack depth to wall thickness ratios (relative depth) $a / t$ and crack aspect ratio ranging from 0.2-0.8 and 0.2-2.0 respectively. Mettu et al. [1992] extended the above work by considering a wider range of cylinder radius to wall thickness ratio $R / t=4,10$ and 300. Kou and Burdekin [2006] further studied the stress intensity factors for circumferential deep surface cracks $(a / t>0.8)$, partly and fully through-wall cracks subjected to axial tension. Predan et al. [2013] presented numerical studies of Modes II and III stress intensity factors of a semi-elliptical surface crack on the cross section of a hollow cylinder subjected to pure torsion loading. As for inclined cracks, Ayhan [2004] obtained stress intensity factors for deflected and inclined surface cracks. However, this study is limited to finite-thickness plates but not for pipes. In addition, only semi-circular surface cracks were considered.

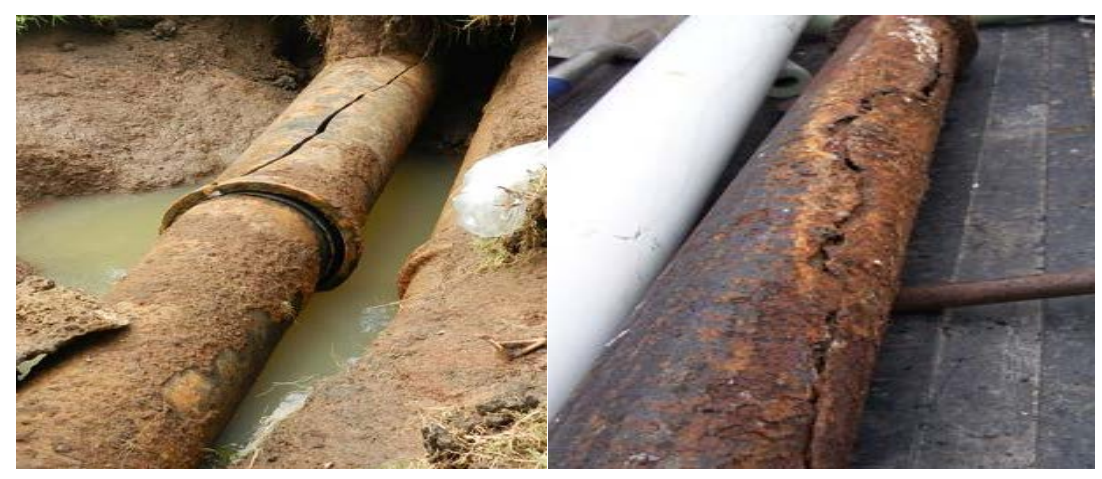

Figure. 1. Failure due to inclined cracks [From URL]

A further literature review suggests that little research has been conducted on stress intensity factors of pressurized pipes with inclined cracks. However, inclined cracks do occur on pipes as shown in figure 1. As investigated by Makar et al. [2001] from exhumed pipes, inclined cracking failures occurred on medium diameter (380-500 mm) pipes. Inclined cracks have also been found on steel pipes due to high $\mathrm{pH}$ (power of Hydrogen) stress corrosion cracking in Australia and Canada [Giggs et al. 2015]. In addition, although some cracks are classified as longitudinal or circumferential ones, it can be observed that they are actually 
inclined or partially inclined cracks. Given such an unacceptably high failure rate of metal pipes [Makar 2000], it is imperative to have a thorough study of every possible cause of failures. Therefore, there is a well-justified need to investigate the stress intensity in pipes with inclined cracks on the external surfaces.

This paper intends to determine the stress intensity factors for external inclined surface cracks in pipes subjected to internal pressure. As the hoop stress induced by internal pressure is not perpendicular to the inclined crack surface, mode mixity exists along the crack front. In this paper, a three-dimensional finite element model is employed to derive the solution to the mixed mode problems. Due to the geometry complexity of the region around inclined cracks, a meshing technique with mixed quadratic tetrahedron and hexahedron elements is employed to simplify the modelling. After verification of the finite element model, the stress intensity factors for inclined cracks are obtained for various parameters of the pipe and cracks including different aspect ratios, inclination angles, relative depths and wall thickness to pipe internal radius ratios.

\section{STRESS INTENSITY FACTORS FOR MIXED MODE CRACKS}

It is well known that for stress analysis of elastic materials, stress singularity exists at the crack region. In order to quantify the stress singularity, a term called stress intensity factor (SIF) $\boldsymbol{K}$ is introduced and it is a function of the applied loads and the cracked geometry including the size, shape and orientation of the crack, and dimensions of the structure where the crack occurs. According to the movements of the upper and lower crack surfaces, the modes of deformation for a cracked body can be classified into three basic modes [Parker 1981]: mode I (the opening mode), mode II (the in-plane shear mode) and mode III (the outof-plane shear mode). When the applied loading is perpendicular to the crack plane, the deformation of the cracked body will be limited to mode I. Otherwise, mode II and/or mode III will also occur.

For cracked pipes, the equation for mode I stress intensity factor at any point along an elliptical or semielliptical crack in Raju and Newman [1982] can be extended for the stress intensity factors with mixed modes for an inclined crack with the consideration of the inclination angles as follows:

$$
\boldsymbol{K}=p R / t \sqrt{\pi a / Q} \boldsymbol{F}(a / t, a / c, t / R, \xi, \theta)
$$

where $\boldsymbol{K}=\left\{\begin{array}{lll}K_{I} & K_{I I} \quad K_{I I I}\end{array}\right\}^{\boldsymbol{T}}, p$ is the internal pressure, $R$ is the internal radius of the pipe, $t$ is the thickness of the pipe, $p R / t$ is "the average" hoop stress for pipes, $a$ is the crack depth, $Q$ is the shape factor for an ellipse and is given by the square of the complete elliptical integral of the second kind, $c$ is half of crack length, $\xi$ is used to define the position along the semi-elliptical crack and $\theta$ is the angle between the crack and the longitudinal direction as shown in figure 2. When $\theta$ equals to 0 , the crack is a longitudinal crack and when $\theta$ equals to $90^{\circ}$, the crack is a circumferential crack. $\boldsymbol{F}(a / t, a / c, t / R, \xi, \theta)=\left\{\begin{array}{lll}F_{I} & F_{I I} & F_{I I I}\end{array}\right\}^{T}$ is the influence coefficient function.

Analytical expressions of stress intensity factors with mixed modes are only available for some simple problems with cracks. For most problems, numerical approximations have to be resorted to. There are two methods that can be used to evaluate the stress intensity factors with mixed modes: one is based on displacement while the other is based on energy. The displacement method requires very fine meshing around the crack region for acurate analysis. In comparison, the energy based $J$ integral method is theoretically path-independent and can produce accurate results with relatively coarse meshes. Therefore, the energy based $J$ integral is employed in this study to determine the stress intensity factors.

In three dimensions, the $J$ integral can be expressed as follows [Shih et al. 1986]:

$$
J=\lim _{\Gamma \rightarrow 0} \int_{\Gamma}\left(\kappa \delta_{1 i}-\sigma_{i j} u_{j, 1}\right) n_{i} d \Gamma
$$

where $\kappa$ is the elastic strain energy density, $i$ and $j$ range from 1 to $3, \delta_{1 i}$ is the Kronecker delta, $\sigma_{i j}$ is the 
stress components, $u_{j}$ represents the displacement components, $u_{j, 1}=\partial u_{j} / \partial x_{1}$ and $n_{i}$ is the unit vector perpendicular to the boundary $\Gamma$. When all three types of fracture modes exist, the $J$ integral equals to the energy release rate $G_{e}$ for linear elastic porblems, given by [Bowness and Lee 1995]:

$$
J=G_{e}=\frac{K_{I}^{2}}{E^{\prime}}+\frac{K_{I I}^{2}}{E^{\prime}}+\frac{(1+v) K_{I I I}^{2}}{E}
$$

where for plane strain problems $E^{\prime}$ needs to be replaced by $E /\left(1-v^{2}\right)$ while for plane stress problems, $E^{\prime}=E$. In order to calculate these three stress intensity factors, interaction integral methods seem to be the most accurate method [Waters et al. 2005]. In these methods, auxiliary crack fields with pure mode I, pure mode II and mode III are required and each auxiliary field is superimposed on the actual crack field to obtain three sets of equations [Shih and Asaro 1988].
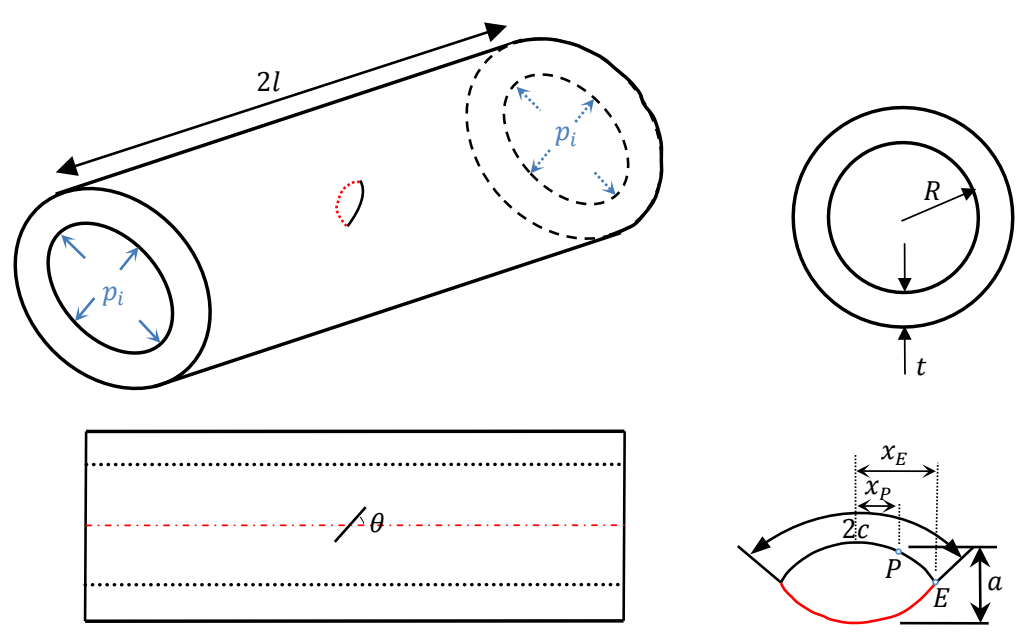

Figure 2. A pressurized pipe with an inclined external surface crack

\section{THREE-DIMENSIONAL FINITE ELEMENT MODELLING}

In this study, a widely used finite element software ABAQUS [2011] is employed to conduct the numerical analyses. Consider a pipe with an inclined crack as shown in figure 2. The internal radius of the pipe is $R$, the thickness of the pipe wall is $t$, the crack depth is $a$, the crack length is $2 c$ and the whole pipe length is $l$. The Poisson's ratio is taken to be 0.3 for all pipe analyses. The quarter-point elements are utilized around the crack front in order to produce a square-root singularity of stress and strain fields at the vicinity of the crack tip. A small volume called the "crack tunnel" containing the wedge elements is created. Due to the complexity of the mesh around the crack region, especially for the inclined cracks on the external surface of the pipe, a meshing technique with quadratic elements of mixed types is employed to conduct the fracture analysis, which simplifies the geometric modelling of the cracked pipes considerably. The irregular region close to the crack region is meshed with quadratic tetrahedron elements while the other regions are divided by quadratic hexahedron elements. Tie constraints are applied on the interfaces between the tetrahedron elements region and the hexahedron elements regions.

Before determining the influence coefficients for inclined external surface cracks in pipes, it is essential to verify the accuracy of the above 3D FEM model. First, the results of influence coefficients of the stress intensity factors for longitudinal external cracks in pipes by Raju and Newman [1982] are used for comparison. The whole pipe models are created with semi-circular external surface cracks of different relative depth ratios. In each model, the nodes with minimum Y value in the pipe model are fixed and the 
internal pressure is applied to the internal surface of the pipe directly. One whole pipe model with a longitudinal crack is shown in figure 3 (a). The region surrounding the crack tunnel is meshed with tetrahedron elements (figure 3 (b)), while the other parts are meshed with hexahedron elements with the layer of elements in the crack tunnel (figure 3 (c)) close to the crack front collapsed to wedge elements (figure 3 (d)). In this comparison, $\xi$ is the same as $\varphi$ in Raju and Newman [1982] to define the positions of the points along the crack front. As shown in Table 1, the influence coefficients of the mode I stress intensity factors are calculated and compared with those determined by the following equation from Raju and Newman [1982]:

$$
F_{e}=\frac{t}{R}\left(\frac{R^{2}}{R_{0}^{2}-R^{2}}\right)\left[2 G_{0}+2\left(\frac{a}{R_{0}}\right) G_{1}+3\left(\frac{a}{R_{0}}\right)^{2} G_{2}+4\left(\frac{a}{R_{0}}\right)^{3} G_{3}\right]
$$

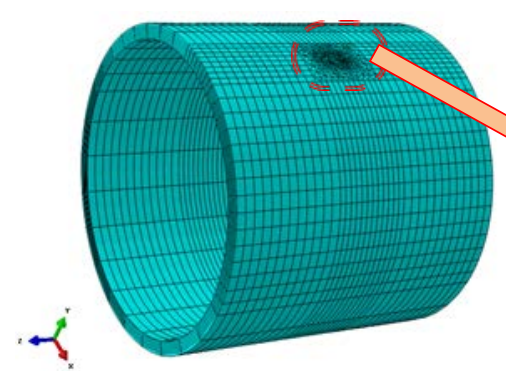

(a) Global view of cracked pipe

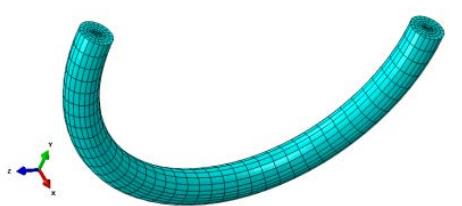

(c) Crack tunnel with hexahedron elements

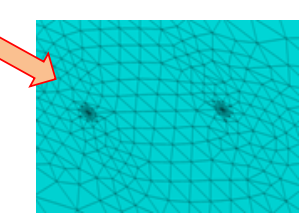

(b) Close-up view of the crack region

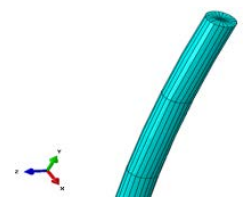

(d) Wedge elements around the crack tip

Figure 3. Finite element model of a whole pipe with a longitudinal crack

Table 1. Comparison of influence coefficients $F_{e}$ with those of Raju and Newman [1982] $(a / c=1.0$ and $t / R=0.1)$

\begin{tabular}{|c|c|c|c|c|c|c|c|c|c|}
\hline \multirow{2}{*}{$\mathbf{2} \boldsymbol{\xi}$} & \multicolumn{3}{|c|}{$\boldsymbol{a} / \mathbf{t}=\mathbf{0 . 2}$} & \multicolumn{3}{c|}{$\boldsymbol{a} / \boldsymbol{t}=\mathbf{0 . 5}$} & \multicolumn{3}{c|}{$\boldsymbol{a}=\mathbf{0 . 8}$} \\
\cline { 2 - 10 } & $\begin{array}{c}\text { Present } \\
\boldsymbol{\pi}\end{array}$ & $\begin{array}{c}\text { Raju and } \\
\text { rewman } \\
{[1982]}\end{array}$ & Difference & $\begin{array}{c}\text { Present } \\
\text { results }\end{array}$ & $\begin{array}{c}\text { Raju and } \\
\text { Newman } \\
{[1982]}\end{array}$ & Difference & $\begin{array}{c}\text { Present } \\
\text { results }\end{array}$ & $\begin{array}{c}\text { Raju and } \\
\text { Newman } \\
{[1982]}\end{array}$ & Difference \\
\hline 0 & 1.105 & 1.104 & $0.03 \%$ & 1.228 & 1.216 & $0.94 \%$ & 1.411 & 1.405 & $0.43 \%$ \\
\hline 0.25 & 1.058 & 1.037 & $2.00 \%$ & 1.154 & 1.127 & $2.50 \%$ & 1.289 & 1.248 & $3.32 \%$ \\
\hline 0.5 & 1.024 & 1.004 & $2.04 \%$ & 1.105 & 1.079 & $2.41 \%$ & 1.170 & 1.131 & $3.35 \%$ \\
\hline 0.75 & 1.014 & 0.992 & $2.21 \%$ & 1.083 & 1.063 & $1.89 \%$ & 1.152 & 1.125 & $2.38 \%$ \\
\hline 1 & 1.011 & 0.989 & $2.29 \%$ & 1.076 & 1.060 & $1.51 \%$ & 1.147 & 1.123 & $2.16 \%$ \\
\hline
\end{tabular}

It can be seen from Table 1 that very good agreement has been achieved. The maximum difference is 3.35\%. This comparison suggests that the influence coefficients obtained from the proposed three-dimensional finite element model are accurate and reliable.

As a further verification, an embedded inclined circular crack in an infinite body under far-field tensile loading is analysed. This problem has also been analysed by Nikishkov and Atluri [1987] and Ayhan [2004] using the equivalent domain integral method and the enriched finite element method respectively. The analytical solution to this problem was given in Murakami [1987] and can be expressed as follows when the inclination angle $\theta=30$ : 


$$
\begin{aligned}
& K_{I}=\frac{3}{2} \sigma \sqrt{\frac{a}{\pi}} \\
& K_{I I}=\frac{\sqrt{3}}{2-v} \sigma \sqrt{\frac{a}{\pi}} \cos \xi \\
& K_{I I I}=\frac{\sqrt{3}(1-v)}{2-v} \sigma \sqrt{\frac{a}{\pi}} \sin \xi
\end{aligned}
$$

where $\xi$ is the angle for a particular point on the crack front and $\sigma$ is the far-field tensile stress.

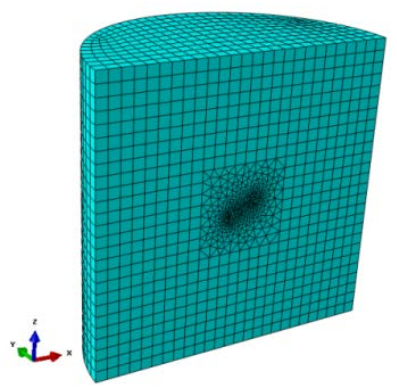

\section{Figure 4. Finite element mesh for half rod model with an inclined crack}

It should be noted that the analytical solution to the mode II and III stress intensity factors used in Nikishkov and Atluri [1987] ignored the Poisson's ratio $v$, i.e., $v=0$ was used. Therefore, the exact values of the modes II and III stress intensity factors in the figures used for comparison are larger than their actual values. This problem is analysed in this paper using the finite element method with the energy based $J$ integral method by considering a cylindrical rod with $a / R=0.1$ ( $R$ is the radius of the rod) containing an inclined circular crack in its centre. The inclination angle of the crack is $30^{\circ}$, and the height of the rod is taken to be twice of the rod radius. Due to symmetry, only half of the rod is modelled as shown in figure 4 . The symmetry boundary condition is allocated to the $\mathrm{XZ}$ plane. One node at the bottom of the model is also fixed to prevent rigid body rotation. The uniform tensile stress is applied to both the top and bottom of the model. In addition, unit values are used for the crack size and applied tensile stress.

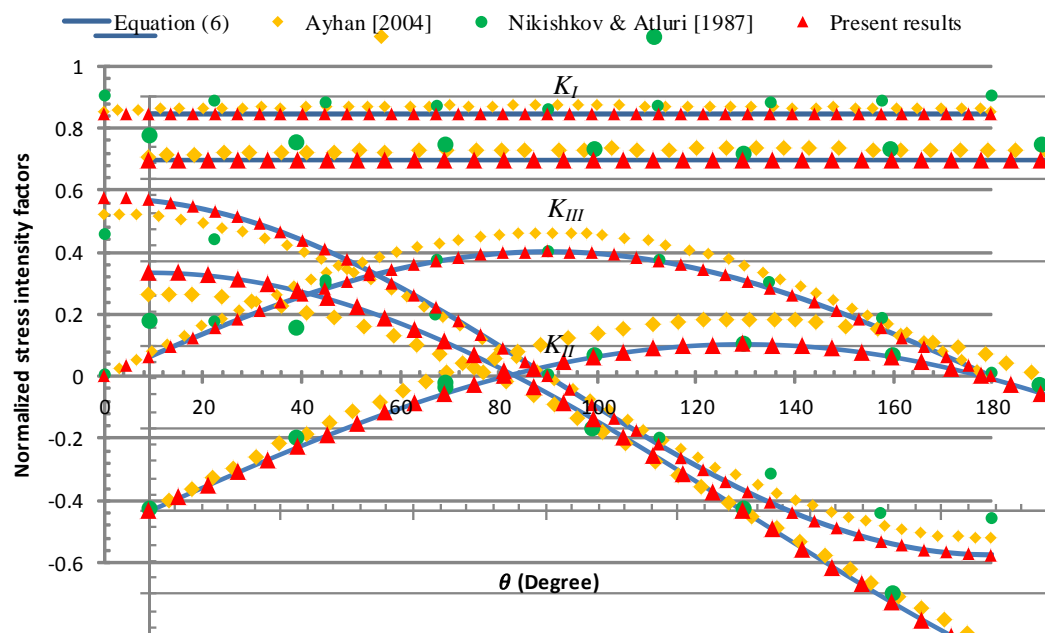

Figure 5. Comparison of stress intensity factors with mixed modes

The analysed results of the stress intensity factors with mixed modes are plotted against the analytical results from Equation (6) together with the results from Nikishkov and Atluri [1987] (s-function (b)) and Ayhan [2004], which were normalized by $\sigma \sqrt{a}$. It can be seen from figure 5 that the analysed results in the present study are in better agreement with the analytical solution and the maximum difference is $1 \%$. 
For the inclined external surface cracks in pipes, only the cases in which the inclined crack is perpendicular to the pipe surface are considered in the present study. The values of the geometry parameters $(a / c, a / t$, $t / R, \xi$ and $\theta$ ) for the inclined surface cracks are given in Table 2. Convergence studies are conducted to obtain the desired finite element mesh for the pipe models with inclination angle $\theta=0$ and the same meshes are used for the models with the same crack and pipe geometries but different inclination angles. The pipe length $l$ is selected to be large enough to reduce its effect on stress intensity factor $(l / c \geq 10$ and $l / a \geq$ $10)$. At any point along the crack line, a normalized coordinate $\xi$ is defined as $\xi=x_{P} / x_{E}$, where $x_{P}$ and $x_{E}$ are defined in figure 2 .

Table 2. Range of geometrical parameters

\begin{tabular}{|c|c|}
\hline Parameter & Range \\
\hline $\boldsymbol{\theta}$ & 0154575 \\
\hline$\frac{\boldsymbol{a}}{\boldsymbol{t}}$ & 0.20 .50 .8 \\
\hline $\boldsymbol{a}$ & 0.41 .01 .5 \\
\hline $\boldsymbol{c}$ & 0.10 .25 \\
\hline $\boldsymbol{\boldsymbol { t }}$ & {$\left[\begin{array}{ll}-1 & 1\end{array}\right]$} \\
\hline
\end{tabular}

\section{RESULTS AND DISCUSSIONS}

It has been known that the order of the stress singularity at the point where the crack front meets the free surface of the pipe depends on the Poisson's ratio $v$ and the intersection angle (Pook 1993). During numerical calculations, non-convergence of the $\mathrm{J}$ integral and mixed-mode stress intensity factors at the surface point of the crack front has been observed. This is attributed to the non-orthogonal intersection of the crack front with the free surface, which results in the loss of path dependence of the $J$ integral. Due to curved pipe surface, at the surface point, there exists a complex stress and strain state, leading to the nonconvergence of the stress intensity factors. In order to more accurately capture the singular behaviour near the free surface, very fine mesh was adopted along the crack front, which was divided into 80 segments for quadratic elements or even more for some cases, and the stress intensity factors with mixed modes at the surface points are estimated by extrapolation from the results near the surface.

A close inspection on the mode I influence coefficient data along the crack front for each case reveals that the maximum values for semi-elliptical cracks with high aspect ratios and semi-circular cracks all take place at the near surface points rather than the surface point. This is attributed to the free surface effects caused by different stress conditions at the surface points (plane stress) and the other points along the crack front (plane strain). Consequently, this leads to different singularity at the free surface points, affecting the strain energy and then the stress intensity factors.

The influence coefficients of stress intensity factors with mixed modes for $a / c=1, t / R=0.1, a / t=0.5$ and different inclination angles are shown in figure 6 for demonstration purposes. From all analysed cases, it can be observed that mixed modes exist along the crack front when an inclined crack $(\theta \neq 0)$ is subjected to internal pressure. At the deepest points of the cracks, only mode I and mode III occur while at the other 
points all three modes take place.

The results show that in all cases the influence coefficients of mode I stress intensity factors are symmetric with respect to the deepest point and decrease along the whole crack front with the increase of the inclination angle $\theta$ (figure 6 (a) and figure 7). It can also be seen that different from the semi-elliptical cracks with low aspect ratio $(a / c<1)$, the semi-elliptical cracks with high aspect ratios exhibit distinct behaviours. As can be seen from figure 7 , the maximum mode I stress intensity factors for cracks with high aspect ratio appear near the free surface (figure 7 (a)) rather than at the deepest point for low aspect ratio crack (figure 7 (b)). For mode II stress intensity factors, the influence coefficients in all cases are anti-symmetric with respect to the deepest point, which can be seen in figure 6 (b). Moreover, when the inclination angle $\theta=45^{\circ}$, the influence coefficients along the whole crack front reach the maximum. In addition, as expected, it can be seen that the influence coefficients for cracks with $\theta=15^{\circ}$ are the same as those for $\theta=75^{\circ}$. In terms of mode III stress intensity factors, the influence coefficients are symmetrical about the deepest point, which is similar to the mode I stress intensity factors. Same as the mode II stress intensity factors, the influence coefficients for $\theta=15^{\circ}$ are the same as those of $\theta=75^{\circ}$, and they take maximum values along the whole crack front when $\theta=45^{\circ}$.

For linear elastic fracture mechanics, an equivalent stress intensity factor $K_{e q}$ can be determined as a function of the three fracture deformation modes $\left(K_{I}, K_{I I}\right.$ and $\left.K_{I I I}\right)$ for plane strain problems as follows [Socie et al. 1987]:

$$
K_{e q}=\sqrt{J E /\left(1-v^{2}\right)}=\sqrt{K_{I}^{2}+K_{I I}^{2}+K_{I I I}^{2} /(1-v)}
$$

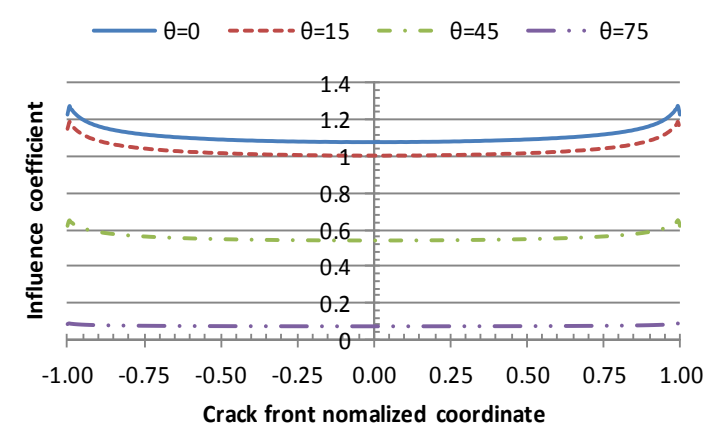

(a) Mode I

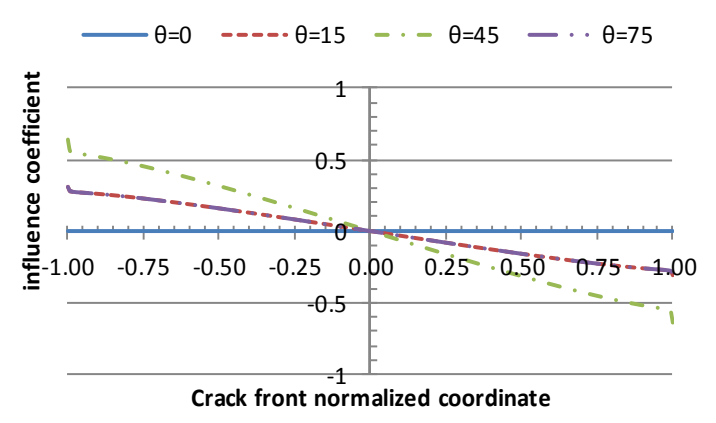

(b) Mode II

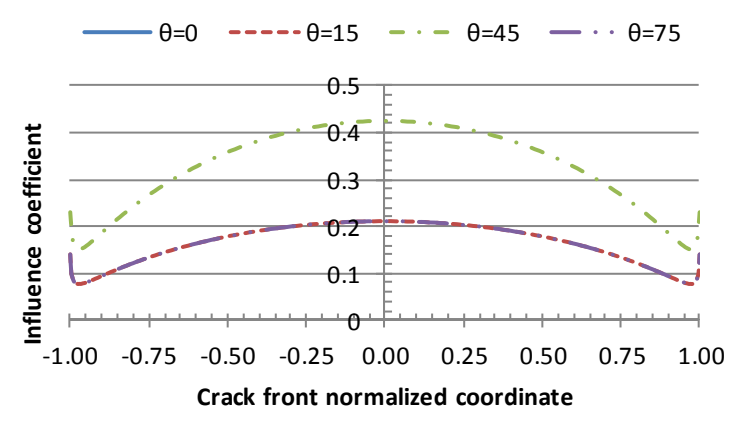

(c) Mode III

Figure 6. Influence coefficients of stress intensity factors with mixed modes $(t / R=0.1$,

$$
a / c=1.0, a / t=0.5)
$$




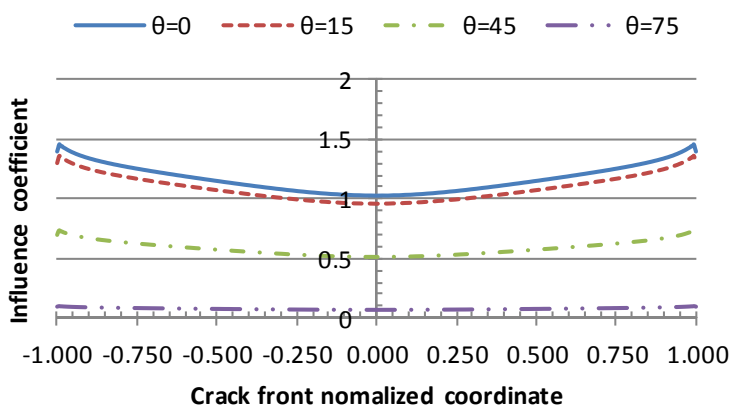

(a) $a / c=1.5$

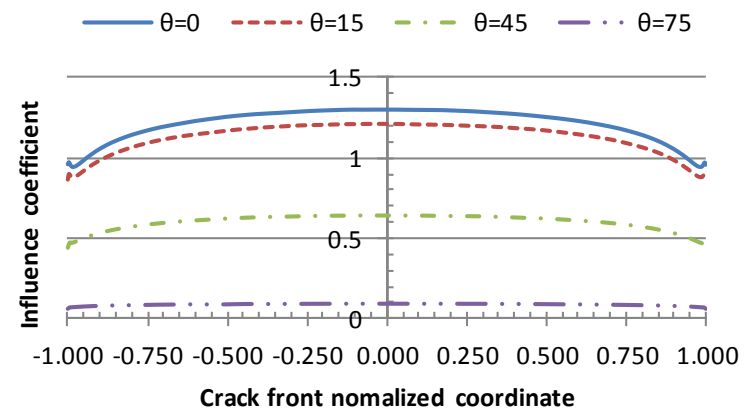

(b) $a / c=0.4$

Figure 7. Mode I influence coefficients for inclined external surface cracks $(t / R=0.1$, $a / t=0.5$ )

Using Equation (7), the influence coefficients of equivalent stress intensity factors are plotted against those of the mode I, II and III stress intensity factors in figure 8 for an inclined external surface crack with $t / R=$ $0.1, a / t=0.5, \theta=45$. The influence coefficients of the equivalent stress intensity factors are found to be symmetric with respect to the deepest point along the crack front and decrease as the inclination angle increases. For given wall thickness to internal radius ratio $t / R$ and aspect ratio $a / c$ and with the increase of the relative depth $a / t$, the influence coefficients for all three modes stress intensity factors increase along the whole crack front for all inclination angles. This indicates that the deeper the crack propagates into the pipe wall, the more critical it becomes as the stress intensity factors increase.

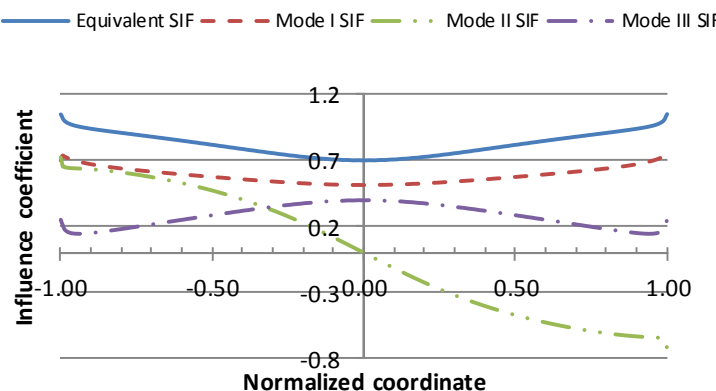

(a) $a / c=1.5$

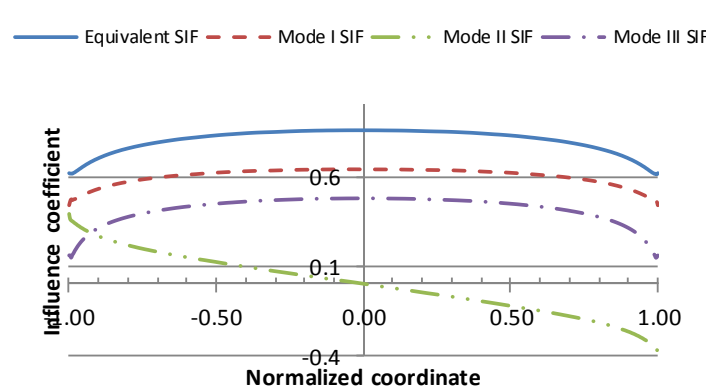

(b) $a / c=0.4$

Figure 8. Influence coefficients of equivalent stress intensity factors and the modes I, II and III components $(t / R=0.1, a / t=0.5, \theta=45)$

\section{CONCLUSION}

In order to study the mixed mode problem of the pressurized pipe with an inclined external surface crack, a three dimensional finite element model with mixed tetrahedron and hexahedron elements is employed. After verification of the proposed finite element model, the influence coefficients of the stress intensity factors for a wide range of inclined external cracks in a pressurized pipe are obtained. It was found that in all the analysed cases, with respect to the deepest point, the influence coefficients of mode I, mode III and equivalent stress intensity factors are symmetric while these of mode II appear to be anti-symmetric. Moreover, with the increase of the inclination angle, the influence coefficients of mode I and equivalent stress intensity factors decrease along the whole crack front. In contrast, those of mode II and mode III reach the maximum when the inclination angle is $45^{\circ}$ and decrease when the angle either increases or 
decrease from $45^{\circ}$. It was also observed that, the semi-elliptical cracks with high aspect ratios $(a / c>1)$ exhibit unique behaviours, which is different from the semi-elliptical cracks with low aspect ratio $(a / c<$ 1), i.e. the maximum mode I intensity factors appears near the free surface rather than at the deepest point. In addition, For given $t / R$ and $a / c$, the influence coefficients of all three modes stress intensity factors along the whole crack front for all inclination angles, increase with the increase of $a / t$.

\section{ACKNOWLEDGEMENT}

Financial support from Australian Research Council under DP140101547 and LP150100413 is gratefully acknowledged.

\section{REFERENCES}

ABAQUS. Version 6.11 (2011). Documentation, Dassault Systèmes Simulia Corp., Providence, RI, USA.

Ayhan, A.O. (2004). "Mixed mode stress intensity factors for deflected and inclined surface cracks in finitethickness plates.” Engineering Fracture Mechanics, 71,1059-79.

Bowness, D., and Lee, M.M.K. (1995). "The development of an accurate model for the fatigue assessment of doubly curved cracks in tubular joints.” International Journal of Fatigue, 73,129-147.

Carpinteri, A., and Brighenti, R. (2000). "A three-parameter model for fatigue behaviour of circumferential surface flaws in pipes.” International Journal of Mechanical Sciences, 42:1255-1269.

Griggs, J., Gamboa, E., and Lavigne, O. (2015). “A review of modelling high pH stress corrosion cracking of high pressure gas pipelines.” Materials and Corrosion, DOI: 10.1002/maco.201508454.

Kou, K.P., and Burdekin, F.M. (2006). "Stress intensity factors for a wide range of long-deep semi-elliptical surface cracks, partly through-wall cracks and fully through-wall cracks in tubular members." Engineering Fracture Mechanics, 73,1693-1710.

Li, C.Q., and Yang, S.T. (2016). "Stress intensity factors for high aspect ratio semi-elliptical internal surface cracks in pipes.” International Journal of Pressure Vessels and Piping, 96-97,13-23.

Lin, X.B., and Smith, R.A. (1997). "Shape growth simulation of surface cracks in tension fatigue round bars.” International Journal of Fatigue, 19(6),461-469.

Lin, X.B., and Smith, R.A. (1998). "Fatigue growth prediction of internal surface cracks in pressure vessels.” Journal of Pressure Vessel Technology ASME, 120,17-23.

Makar, J.M. (2000). “A preliminary analysis of failures in grey cast iron water pipes.” Engineering Failure Analysis, 7(1),43-53.

Makar, J.M., Desnoyers, R., and McDonald, S.E. (2002). Failure modes and mechanisms in gray cast iron pipe. In: Proceedings of NRCC-44218, underground infrastructure research: municipal, industrial and environmental applications, Kitchener, Ontario, 1-10.

Marshall, P. (2001). "The residual structural properties of cast iron pipes-structural and design criteria for linings for water mains.” UK Water Industry Research Ltd., London.

Mettu, S.R., Raju, I.S., and Forman, R.G. (1992). "Stress intensity factor for part-through surface cracks in hollow cylinders." JSC report 25685/LESC report 30124, NASA Lyndon B. Johson Space Center/Lockheed Engineering and Sciences Co. Joint Publication.

Murakami, Y. (1987). “Stress Intensity Factors Handbook.” Pergamon Press. 
Nikishkov, G.P., and Atluri, S.N. (1987). "Calculation of fracture mechanics parameters for an arbitrary three-dimensional crack by the 'equivalent domain integral' method.” International Journal for Numerical Methods in Engineering, 24,1801-1821.

Parker, A.P. (1981). “The mechanics of fracture and fatigue.” Chapman and Hall, London.

Pook, L.P. (1993). “A finite element analysis of the angle crack specimen.” In: Rossmanith, Miller, editors. Mixed mode fatigue and fracture. ESIS, 14. London: Mechanical Engineering Publications, 285-302.

Predan, J., Mocilnik, V., and Gubeljak, N. (2013). "Stress intensity factors (SIF) solutions for circumferential semi-elliptical surface cracks in a hollow cylinder subjected to pure torsion." Engineering Fracture Mechanics, 105,152-168.

Raju, I.S., and Newman, J.G. (1982). "Stress-intensity factors for internal and external surface cracks in cylindrical vessels.” Journal of Pressure Vessel Technology ASME, 104(4),293-298.

Raju, I.S. (1991). "Computations of stress intensity for flaws in flat and cylindrical vessels.” Subcontractor Report to LESC P.O. 02N0140125-1.

Shih, C.F., and Asaro, R.J. (1988). "Elastic-plastic analysis of cracks on biomaterial interfaces: part I-small scale yielding.” Journal of Applied Mechanics-Transactions ASME, 55,299-316.

Shih, C.F., Moran, B., and Nakamura, T. (1986). "Energy release rate along a three-dimensional crack front in a thermally stressed body.” International Journal of Fracture, 30:79-102.

Shiratori, M., Niyoshi, T., and Tanikawa, K. (1987). "Analysis of stress intensity factors for surface cracks subjected to arbitrarily distributed surface cracks.” In: Murakami Y, Editor. Stress intensity factors handbook. US: Pergamon, 698-727.

Socie, D.F., Hua, C.T., and Worthem, D.W. (1987). "Mixed mode small crack growth.” Fatigue \& Fracture of Engineering Materials \& Structrures, 10(1),1-16.

Wang, X., and Lambert, S.B. (1994). "Stress intensity factors and weight functions for longitudinal semielliptical surface cracks in thin pipes.” International Journal of Pressure Vessels and Piping, 65(1),7587.

Waters, M.C., Paulino, G.H., and Dodds, Jr. RH. (2005). "Interaction integral procedures for 3-D curved cracks including surface tractions.” Engineering Fracture Mechanics, 72,1635-1663.

http://www.dailytelegraph.com.au/newslocal/news/burst-water-main-near-goodwin-st-in-west-rydecauses-havoc-this-afternoon/story-fngr8gwi-1226861374547

http://cr4.globalspec.com/thread/22653/Cast-Iron-Sewer-Line-failure 